\title{
Dissecting Carotenoid from Structural Components of Carotenoid-Based Coloration: A Field Experiment with Great Tits (Parus major)
}

\author{
Alain Jacot, ${ }^{1, *}$ Cristina Romero-Diaz, ${ }^{2}$ Barbara Tschirren, ${ }^{3}$ Heinz Richner, ${ }^{3}$ and Patrick S. Fitze ${ }^{2,4}$ \\ 1. Max Planck Institute for Ornithology, Behavioural Ecology and Evolutionary Genetics, Eberhard-Gwinner-Strasse 5, 82319 Seewiesen, \\ Germany; 2. Department of Biodiversity and Evolutionary Biology, Museo Nacional de Ciencias Naturales, Consejo Superior de \\ Investigaciones Científicas, Madrid, Spain; 3. Institute of Ecology and Evolution, University of Bern, Baltzerstrasse 6, 3012 Bern, \\ Switzerland; 4. Instituto Pirenaico de Ecología, Consejo Superior de Investigaciones Científicas, Jaca, Spain
}

Submitted January 8, 2009; Accepted February 25, 2010; Electronically published May 14, 2010

\begin{abstract}
AвSTRACt: Carotenoid-based yellowish to red plumage colors are widespread visual signals used in sexual and social communication. To understand their ultimate signaling functions, it is important to identify the proximate mechanism promoting variation in coloration. Carotenoid-based colors combine structural and pigmentary components, but the importance of the contribution of structural components to variation in pigment-based colors (i.e., carotenoid-based colors) has been undervalued. In a field experiment with great tits (Parus major), we combined a brood size manipulation with a simultaneous carotenoid supplementation in order to disentangle the effects of carotenoid availability and early growth condition on different components of the yellow breast feathers. By defining independent measures of feather carotenoid content (absolute carotenoid chroma) and background structure (background reflectance), we demonstrate that environmental factors experienced during the nestling period, namely, early growth conditions and carotenoid availability, contribute independently to variation in yellow plumage coloration. While early growth conditions affected the background reflectance of the plumage, the availability of carotenoids affected the absolute carotenoid chroma, the peak of maximum ultraviolet reflectance, and the overall shape, that is, chromatic information of the reflectance curves. These findings demonstrate that environmentinduced variation in background structure contributes significantly to intraspecific variation in yellow carotenoid-based plumage coloration.
\end{abstract}

Keywords: structural color, carotenoid-based color, Parus major.

\section{Introduction}

Red, orange, and yellow plumage colors (often referred to as carotenoid-based colors) are important and widespread signals in visual communication in birds (McGraw 2006).

\footnotetext{
* Corresponding author. Present address: Schweizerische Vogelwarte, Aussenstelle Wallis, CH-3970 Salgesch, Switzerland; e-mail: alain.jacot@vogelwarte.ch. Am. Nat. 2010. Vol. 176, pp. 55-62. (c) 2010 by The University of Chicago. 0003-0147/2010/17601-50980\$15.00. All rights reserved. DOI: $10.1086 / 653000$
}

They frequently evolved in a sexual or social context, and their signal content is best understood by conditioncapture models, which predict that signals reliably reveal aspects of an individual's condition or quality (Zahavi 1975; Grafen 1990). These models are based on costs associated with signal expression, where only high-quality individuals can bear the costs of exaggerated ornamentation. The benefits derived from enhanced plumage coloration may include increased sexual attractiveness in mate choice (Hill 2006), increased food provisioning by parents (Tanner and Richner 2008, but see Tschirren et al. 2005), or even reduced risk of predation via enhanced crypsis (Baker and Parker 1979).

Most research on variation in yellow, red, and orange plumage color expression has focused on how the availability of carotenoids translates into variation in coloration and attractiveness. There is growing empirical evidence that carotenoids are often limited and that plumage colors may act as an indicator of quality or condition. For example, great tit nestlings develop a more intense yellow plumage when reared in territories with abundant green butterfly larvae (Eeva et al. 1998). This was confirmed by experimental studies showing that carotenoid supplementation leads to a more intense plumage coloration of nestlings (Fitze et al. 2003; Tschirren et al. 2005; Hadfield and Owens 2006) and adult birds (Shawkey et al. 2006).

However, most if not all colors stem from the combination of a structure that reflects light (i.e., a keratin structure forms the feather and provides it with a background reflectance) and pigments that absorb light at specific wavelengths. Both components contribute to perceived variation in coloration (Shawkey and Hill 2005, 2006; Shawkey et al. 2006). Carotenoid-based feather colors are the result of these two components. First, in many passerines the keratin-based background structure shows a uniform reflectance after an increase in the short wave- 
lengths and thus appears white, thereby determining the overall achromatic reflectance of the feather. Second, carotenoid pigments, which are incorporated into the feather matrix, mainly absorb light in short to middle wavelengths (i.e., violet-blue wavelengths) and determine the chromatic component of a feather. The more carotenoids are incorporated into a feather, the less light is reflected in the violetbluish wavelength, which leads to more long-wavelengthbiased reflectance spectra and thus to a more intense yellow to red coloration. Reflectance spectra of carotenoid-based colors therefore show a pronounced reflectance peak in the ultraviolet (UV) wavelength (between 300 and $400 \mathrm{~nm}$ ), low reflectance in short to middle wavelengths (around 450 $\mathrm{nm}$ ) because of the light-absorbing properties of carotenoid pigments, and a high, plateaulike reflectance curve in the long waveband (yellow to red wavelengths between 550 and $700 \mathrm{~nm}$; fig. 1). Thus, variation in shape and overall reflectance of the spectra indicate that different, potentially independent mechanisms determine coloration. To date, we have very limited knowledge of the relative contribution of the background structure and pigment content on plumage coloration and what environmental and genetic factors determine this variation.

In a recent study on captive American goldfinches, Shawkey et al. (2006) tried to unravel the extrinsic factors that contribute to variation in structural and pigmentbased color components of carotenoid-based coloration and concluded that variation in the yellow color is mainly pigment based. However, the outcome may depend on differences between species in resource allocation tradeoffs affecting plumage coloration, in life-history stages, and in environment, and it may depend on the applied methodology. Consequently, more studies are necessary to understand factors affecting variation in structural components of carotenoid-based colors.

The aim of this study is to disentangle the effects of two biologically relevant environmental factors-carotenoid availability and early growth condition-on the background structure and the carotenoid pigments, both affecting feather coloration. In a field experiment in great tit nestlings (Parus major), we simultaneously manipulate brood size (i.e., early growth conditions) and carotenoid availability and investigate their effects on the color expression of the breast plumage, by using spectral measurements that allow independent measures of carotenoidbased and structural color components. This experimental design thus allows investigation of the absolute and relative contribution of two environmental factors on structural and pigment-based components of yellow plumage colors, in both the human-visible and the UV ranges.
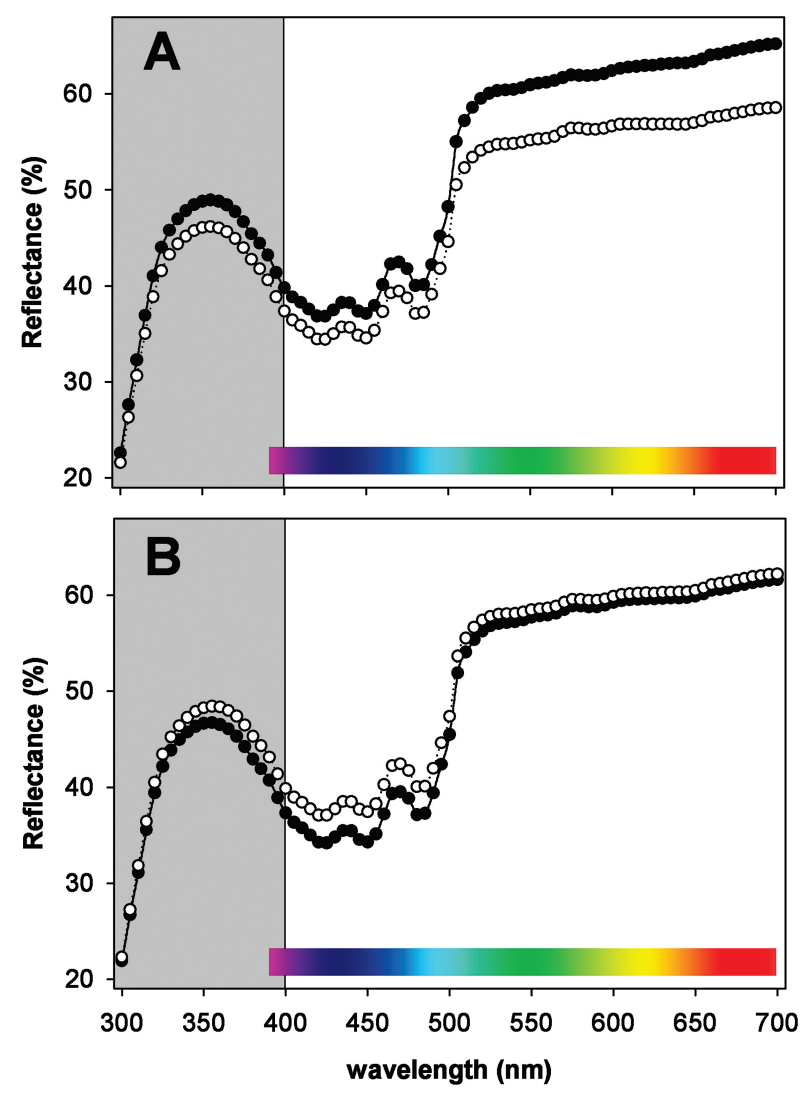

Figure 1: Reflectance spectra from the breast feathers of great tit nestlings across the range of spectral sensitivity in relation to the brood size manipulation $(A)$ and the carotenoid supplementation $(B)$. Spectra with open symbols indicate chicks in the control treatments, whereas filled symbols indicate the mean spectra of reduced-brood and carotenoid-fed chicks in $A$ and $B$, respectively.

\section{Methods}

The field study was conducted in 1999 in a great tit population breeding in nest boxes in a mixed deciduous forest close to Bern, Switzerland (Forst: $46^{\circ} 54^{\prime} \mathrm{N}, 7^{\circ} 17^{\prime} \mathrm{E}$; $\left.46^{\circ} 57^{\prime} \mathrm{N}, 7^{\circ} 21^{\prime} \mathrm{E}\right)$. Nest boxes were checked regularly for start of egg laying, start of incubation, and hatching.

This study is partly based on an experiment that has been published elsewhere (Tschirren et al. 2003). The published results showing effects of carotenoid supplementation and brood size manipulation on plumage coloration were based on digital photography. This method involves the human-visible range (400-700 nm) only. It (1) excludes effects in the ultraviolet part (300-400 nm) of the spectrum and (2) is unable to make conclusions about the relative importance of background coloration and carotenoid content on variation in plumage coloration. Here, we present new results using spectrophotometric mea- 
surements, which offer the possibility to get insight into the mechanism involved in plumage color expression (Shawkey et al. 2006). Additionally, we use numerical simulations to investigate the effects of carotenoid supplementation on ultraviolet signals, which have received growing attention in recent years (e.g., Siefferman and Hill 2005; Shawkey et al. 2006; Jacot and Kempenaers 2007), and we validate the obtained results using a carotenoid removal experiment.

\section{Brood Size Manipulation}

The brood size manipulation (BSM) and partial crossfostering experiment is explained in detail by Tschirren et al. (2003). To summarize, nestlings were exchanged among pairs of nests with the same hatching date and similar brood size in order to create reduced $(-2)$ and naturally sized broods. This was realized by (1) replacing one egg with an artificial egg during the incubation period and (2) simultaneously conducting a brood size manipulation with a partial cross-fostering on day 2, where one extra chick was moved from reduced to enlarged broods. After the brood size manipulation, nests from the control and reduced broods contained a similar number of cross-fostered, unrelated, and own chicks (repeated-measures ANOVA with number of cross-fostered and number of own chicks as repeated measures; $F=0.22$, $\mathrm{df}=1,49, P=.64)$ and significantly different numbers of nestlings $(F=23.82$, $\mathrm{df}=1,49, P<.01)$. The brood size manipulation significantly affected nestling growth rate (see Tschirren et al. 2003).

\section{Carotenoid-Feeding Treatment}

Within one nest, half of the nestlings were fed with either carotenoid beadlets or beadlets containing no carotenoids. Chicks were fed six times every other day, starting 3 days posthatching (i.e., on days 3, 5, 7, 9, 11, and 13). Nestlings from the experimental group received carotenoid beadlets (17 $\pm 0.25 \mathrm{mg})$ containing $5.58 \%$ lutein and $0.44 \%$ zeaxanthin, whereas nestlings of the control group were fed with placebo beadlets ( $17 \pm 0.25 \mathrm{mg}$ ) without carotenoids. The lutein/zeaxanthin ratio in the carotenoid beadlets and the quantity of ingested carotenoids were similar to the amounts found in the natural diet of great tit nestlings (Partali et al. 1987). For a more detailed description on the carotenoid-feeding treatment, see Tschirren et al. (2003).

\section{Measurement of Plumage Coloration}

We used a handheld spectrometer (AvaSpec-2048, Avantes, Eerbek, the Netherlands) connected to a deuterium- halogen light source (AvaLight-DHS, Avantes, Eerbek, the Netherlands) through a bifurcated fiber-optic probe. The probe was fitted at the end with a plastic cylinder to standardize measuring distance and exclude ambient light, and it was held perpendicular to the surface of the feathers. In the field, we plucked breast feathers in a standardized way from each nestling on day 16 posthatching, in order to measure variation in the yellow feather coloration. In the laboratory, five breast feathers were laid on top of each other and measured against a black velvet background with uniform, low reflectance across all wavelengths. Reflectance spectra were measured on five spots on the top of the erupting breast feather. Total feather length and the length of the erupted part of the feather were measured with slide calipers. The experimental treatments did not influence feather length (generalized linear mixed model [GLMM] with Gaussian error distribution and box as a random intercept; sex: $t_{329}=0.48, \quad P=.63$; BSM: $t_{329}=0.15, P=.88$; carotenoids: $\left.t_{329}=0.07, P=.94\right)$ or percentage of erupted feathers (GLMM with Gaussian error distribution and box as a random intercept; sex: $t_{329}=0.37, P=.71$; BSM: $t_{329}=-0.01, P=.99$; carotenoids: $t_{329}=0.79, P=.43$ ), and the treatment effects did not change when adding absolute or relative feather length into the models.

Feather reflectance was calculated between 300 and 700 $\mathrm{nm}$, relative to a WS-2 white standard (Avantes, Eerbek, the Netherlands). We computed indexes, which describe the white background structure and the carotenoid content of the feather (modified from Shawkey et al. 2006). First, we measured background reflectance, which is the absolute reflectance between 575 and $700 \mathrm{~nm}$ and describes the white background structure. These wavelengths are above the absorption properties of carotenoids, and background reflectance thus represents achromatic color brightness (Andersson and Prager 2006). Note that Shawkey et al. (2006) used a similar measure called yellow chroma. However, they used the proportion of light reflecting between 575 and $600 \mathrm{~nm}$ (i.e., $R_{575-600 \mathrm{~nm}} / R_{320-700 \mathrm{~nm}}$ ). By taking the proportion, their measure depends on the amount of carotenoids incorporated into the feather and is therefore not predicted to be an accurate measure of structure-related background reflectance. Second, we measured violet-blue chroma, which is the percentage of total light reflected between 400 and $515 \mathrm{~nm}$ (i.e., $R_{400-515 \mathrm{~nm}} / R_{300-700 \mathrm{~nm}}$; Shawkey et al. 2006). Carotenoids absorb light at these short to middle wavelengths (see fig. $2 B$ ), and the light reflected between 400 and $515 \mathrm{~nm}$ has been shown to be a good indicator of the quantity of carotenoids (lutein, zeaxanthin) incorporated into bird feathers. Third, we defined another measure of carotenoid content called absolute carotenoid chroma. Given that the total amount of light reflected also depends on the amount of carotenoids in- 


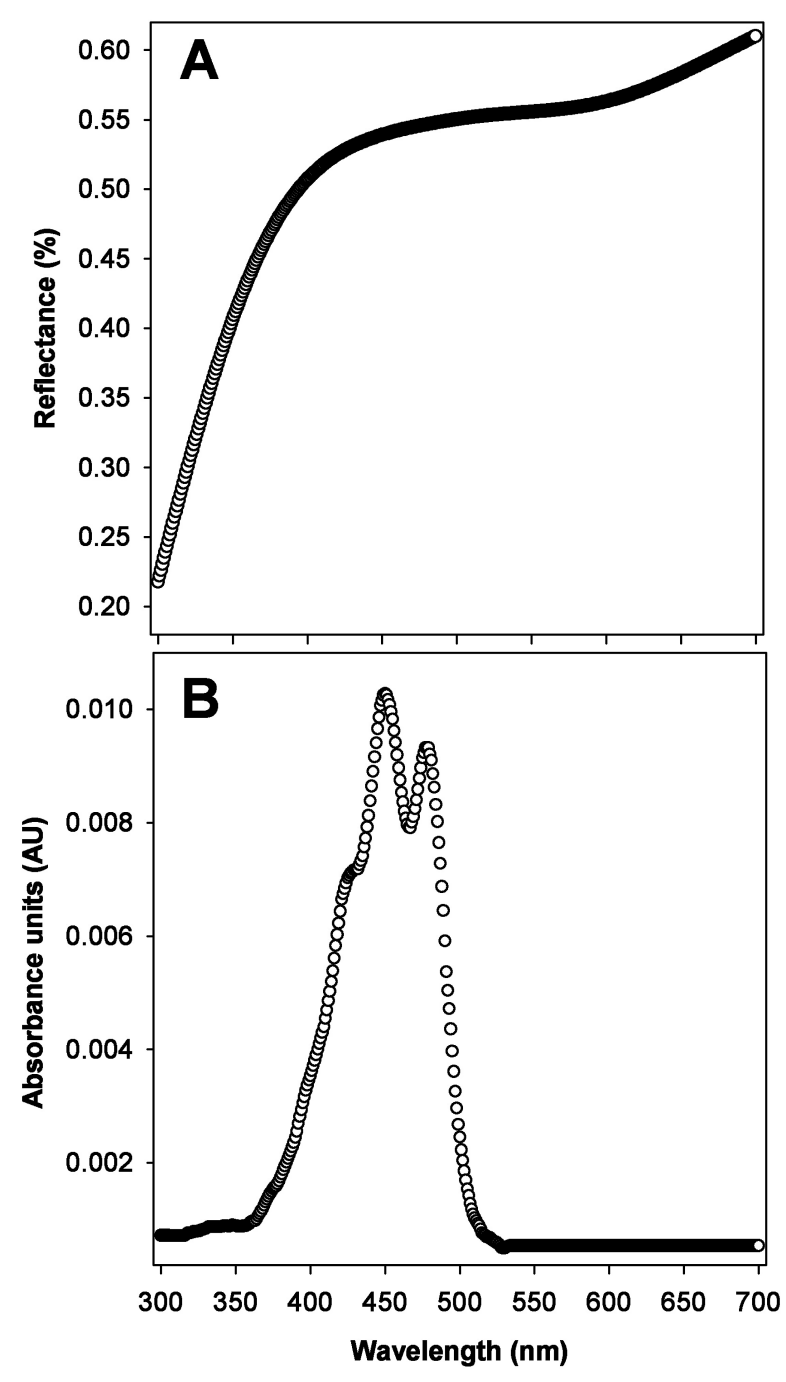

Figure 2: Mean reflectance spectra of the breast feather's background structure $(A)$ and absorption spectra of the carotenoids $(B)$ of six nestling great tits.

corporated (the fewer carotenoids incorporated, the bigger $R_{400-515 \mathrm{~nm}}$ and thus the bigger $R_{300-700 \mathrm{~nm}}$ ), we defined a new variable called absolute carotenoid chroma that takes into account variation in $R_{400-515 \mathrm{~nm}}$ due to differences in the background structure (i.e., $R_{400-515 \mathrm{~mm}} / R_{575-700 \mathrm{~nm}}$ ). Both violet-blue chroma and absolute carotenoid chroma are significantly correlated with another measure of carotenoid content named carotenoid chroma, $\left(R_{700}-R_{450}\right) / R_{700}$, which has been used in other studies (e.g., Peters et al. 2004; Jacot and Kempenaers 2007). Finally, we computed two different indexes for UV reflectance. We measured the wavelength of peak reflectance in the ultraviolet part $\left(R_{\text {UVpeak }}\right)$ and UV chroma as the proportion of light re- flecting in the UV while controlling for background reflectance (i.e., $R_{300-400 \mathrm{~nm}} / R_{575-700 \mathrm{~nm}}$ ).

\section{Simulation Study}

To understand whether and how carotenoid incorporation affects UV reflectance, we conducted a numerical simulation with basic data obtained from nestling breast feathers originating from the same study site. To determine the reflectance of the background structure (fig. $2 A$ ), we removed the carotenoids from the feathers of six unrelated and untreated nestlings. We placed five feathers in glass tubes, added $2 \mathrm{~mL}$ of acidified pyridine, and incubated the solution for $3 \mathrm{~h}$ at $95^{\circ} \mathrm{C}$ (Shawkey and Hill 2005). Thereafter, feathers were air dried, and their coloration was measured as described above. The extracted carotenoids were analyzed using the high-performance liquid chromatography (HPLC) methods of McGraw and Gregory (2004). From the HPLC analyses, we derived the absorption spectra of the carotenoids present in the feathers (fig. 2B). Means and standard deviation per wavelength $(\lambda)$ were derived from all nestlings used in the experiments and were used for simulating the effects of carotenoid incorporation on UV reflectance. Carotenoid incorporation was simulated according to the following equations:

$$
R_{\lambda}^{T}=R_{\lambda}^{\mathrm{BS}}-A_{\lambda}^{T}
$$

where $R_{\lambda}^{T}$ is the reflectance at wavelength $\lambda$ for the carotenoid treatment $(T)$ with the control and carotenoidtreated levels. Variable $R_{\lambda}^{\mathrm{BS}}$ refers to the reflectance of the background structure (BS), and $A_{\lambda}^{T}$ refers to the absorbance of the carotenoids at $\lambda$ for treatment $T$. Absorbance is related to reflectance as

$$
R=10^{-A}
$$

(Botev 1979).

We performed individual-based simulations, including stochasticity on the amount of carotenoids incorporated for the control and carotenoid-supplemented nestlings and stochasticity on the background structure. We assumed that variability within the background structure and within and between treatment levels follows Gaussian laws and corresponds to the observed variances from the field study and the additional background structure measurements $\left(R_{450}^{\text {control }}, 0.37 \pm 0.09 \mathrm{SD} ; R_{450}^{\text {carotenoid }}, 0.34 \pm 0.91 \mathrm{SD} ; R_{700}^{\mathrm{BS}}\right.$, $0.61 \pm 0.15 \mathrm{SD})$. The simulation is based on 10,000 simulations for the control and carotenoid-supplemented nestlings. 
Table 1: Estimates $( \pm \mathrm{SE}$ ) and test statistics (generalized linear mixed model with Gaussian error distribution and box as a random intercept) for nestling plumage color in relation to brood size manipulation and carotenoid supplementation $(n=348)$

\begin{tabular}{|c|c|c|c|c|}
\hline Factor & Estimate & SE & $t$ & $P$ \\
\hline \multicolumn{5}{|c|}{ Index of background structure, background reflectance $\left(R_{575-700 \mathrm{~nm}}\right)$ : } \\
\hline Intercept & $1,481.20$ & 45.89 & 32.28 & \\
\hline Brood size manipulation $^{a}$ & 145.74 & 63.97 & 2.28 & .02 \\
\hline Carotenoid treatment $\mathrm{t}^{\mathrm{b}}$ & -6.49 & 32.65 & -.20 & .84 \\
\hline \multicolumn{5}{|c|}{ Index of carotenoid content 1 , violet-blue chroma $\left(R_{400-515 \mathrm{~nm}} / R_{300-700 \mathrm{~nm}}\right)$ : } \\
\hline Intercept & .23 & .002 & 110.51 & \\
\hline Brood size manipulation $^{\mathrm{a}}$ & -.0017 & .003 & -.58 & .56 \\
\hline Carotenoid treatment $\mathrm{t}^{\mathrm{b}}$ & -.01 & .0016 & -6.19 & $<.01$ \\
\hline \multicolumn{5}{|c|}{ Index of carotenoid content 2 , absolute carotenoid chroma $\left(R_{400-515 \mathrm{~nm}} / R_{575-700 \mathrm{~nm}}\right)$ : } \\
\hline Intercept & .62 & .01 & 64.09 & \\
\hline Brood size manipulation $^{\mathrm{a}}$ & -.01 & .01 & -.91 & .36 \\
\hline Carotenoid treatment $\mathrm{t}^{\mathrm{b}}$ & -.04 & .03 & -5.76 & $<.01$ \\
\hline \multicolumn{5}{|c|}{ Ultraviolet chroma $\left(R_{300-400 \mathrm{~nm}} / R_{575-700 \mathrm{~nm}}\right)$ : } \\
\hline Intercept & .59 & .01 & 62.95 & \\
\hline Brood size manipulation $^{\mathrm{a}}$ & -.02 & .01 & -1.53 & .13 \\
\hline Carotenoid treatment $\mathrm{t}^{\mathrm{b}}$ & -.02 & .006 & -3.52 & $<.01$ \\
\hline
\end{tabular}

\section{Carotenoid Removal Experiment}

To experimentally prove the results of the simulation study, we performed an experiment where we removed all carotenoids from feathers of 20 randomly selected nestlings, each originating from a different nest. We measured feather reflectance of natural yellow feathers and thereafter removed the carotenoids as described above. Using repeated-measures ANOVAs with spectral measurements of natural yellow and depigmented white feathers, we investigated how carotenoids affect the color parameters.

\section{Statistical Analyses}

All statistical analyses were performed with $\mathrm{R}$, version 2.8.0 (R Development Core Team 2008). To analyze the effects of the experimental treatments on plumage coloration, all models included the brood size manipulation and the carotenoid supplementation as fixed factors and box of origin and rearing as random factors. We performed mixedeffect models with crossed-random effects as described by Baayen et al. (2008) using the lme4 package (Bates and Sarkar 2006). In mixed models with a complex random structure (i.e., more than one random level and independent cross-random effects), the test statistics only approximate a $t$ distribution. Therefore, the denominator degrees of freedom cannot be computed accurately and are at their upper bound (default in R, ver. 2.8.0). The standard model diagnostics of nonnormal errors, nonconstant error variance, and the presence of outliers were performed on each of the final models according to Fox (2002), and all models fulfilled model assumptions.

\section{Results \\ Effects of Early Growth Conditions}

Early growth conditions significantly affected the plumage coloration of nestlings. Chicks growing up in reduced broods had a higher background reflectance, indicating higher reflectance of the white background structure (table 1; figs. 1A, 3A). Violet-blue chroma, absolute carotenoid chroma, wavelength of peak reflectance in the ultraviolet part, and UV chroma were not affected by the brood size manipulation (table 1). In summary, the brood size manipulation affected achromatic plumage reflectance, while chromatic aspects remained unaffected.

\section{Effects of the Carotenoid Supplementation}

The availability of carotenoid pigments strongly affected chromatic aspects of the color, while achromatic parameters remained unaffected. Background reflectance remained unaffected by the carotenoid supplementation (table 1). In contrast, the supplementation of lutein and zeaxanthin affected reflectance properties in the UV part and the short wavelengths. Carotenoid-fed chicks had lower violet-blue chroma and absolute carotenoid chroma values, indicating that more carotenoids had been incorporated into the feathers (table 1; figs. $1 B, 3 B$ ).

Because of the light absorption properties of carot- 


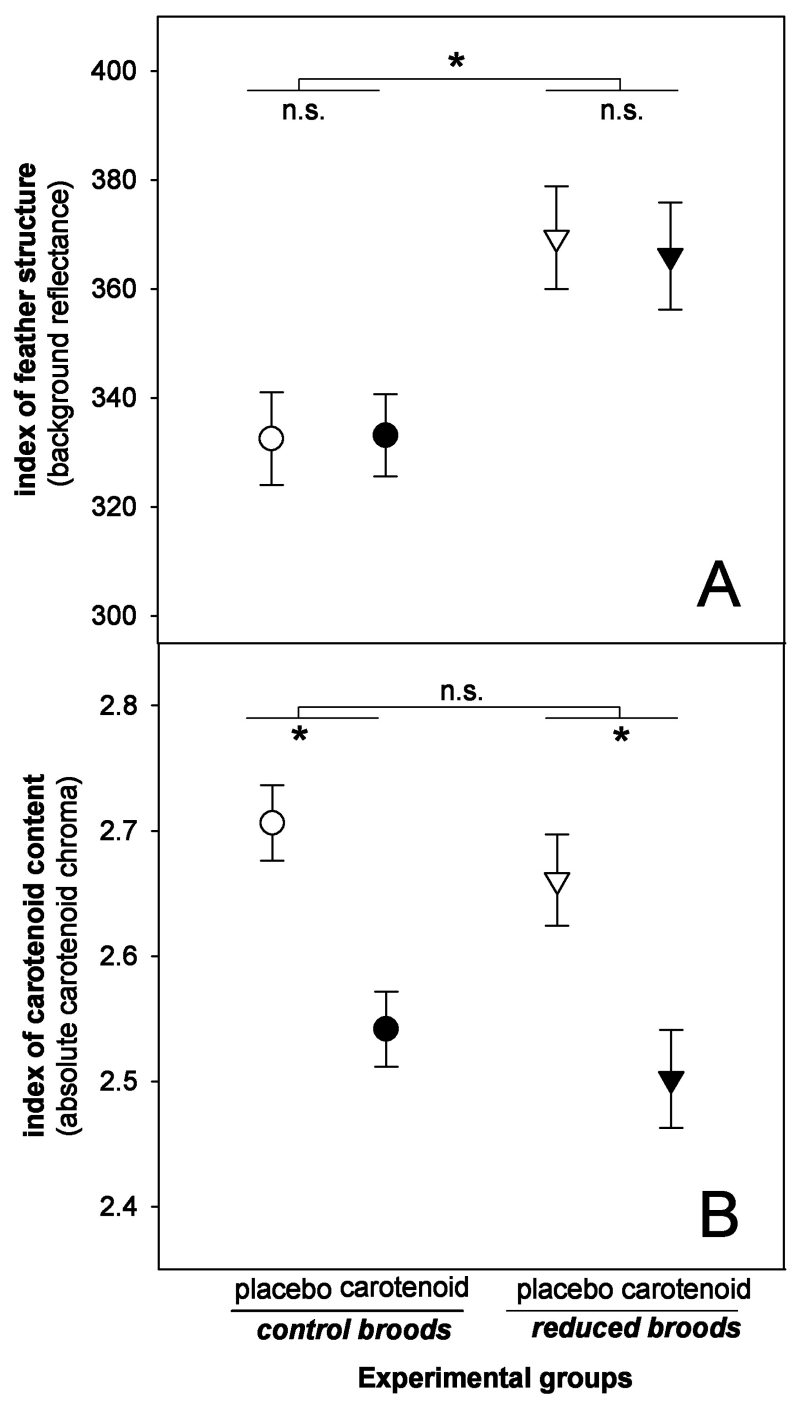

Figure 3: Effects of the brood size manipulation and the carotenoid supplementation on achromatic background reflectance $(A)$ and absolute carotenoid chroma $(B)$. Graphs show means and standard errors of the raw data, whereas the in-text statistics are based on restricted maximum likelihood mixed models.

enoids in the short and middle wavelengths, the reflectance between 300 and $400 \mathrm{~nm}$ (i.e., in the ultraviolet wavelengths) is reduced in carotenoid-fed compared to placebo-fed nestlings (table 1). The carotenoid supplementation $(t=-4.5, n=348, P<.01)$ additionally affected the wavelength with the highest reflectance in the $\mathrm{UV}$, while there were no detectable effects of the brood size manipulation $(t=0.7, n=348, P=.48)$. Carotenoid-supplemented nestlings had UV peaks shifted toward shorter wavelengths (fig. 4).

The numerical simulations show that increased carot- enoid incorporation leads to a shift in the wavelength with the highest reflectance in the ultraviolet. Carotenoidsupplemented nestlings had UV peaks, which were shifted by $1.49 \mathrm{~nm} \pm 0.02 \mathrm{SE}$ toward shorter wavelengths. This corresponds quite nicely to the experimentally induced shift of $1.71 \mathrm{~nm}$ (from $354.31 \pm 0.35$ to $352.60 \pm 0.29$ $\mathrm{nm}$ in carotenoid-fed nestlings), indicating that differences in the UV properties are mainly the consequence of carotenoid incorporation. The simulation further reveals that carotenoid incorporation does not affect the background reflectance measure $(F=2.03, \mathrm{df}=1,19,998$, $P=.15)$ but affects our UV chroma $(F=114,427.3$, $\mathrm{df}=1,19,998, P<.01)$ and absolute carotenoid chroma $(F=112,398.3, \mathrm{df}=1,19,998, \quad P<.01)$ scores. These findings are in line with the experimental results of the carotenoid treatment.

The carotenoid removal experiment confirms the results of the simulation study. The removal of carotenoids affected violet-blue chroma $(F=21.75, \mathrm{df}=1,19, P<$ $.01)$, absolute carotenoid chroma $(F=176.79, \mathrm{df}=$ $1,19, P<.01)$, UV peak reflectance $(F=1,165.47, \mathrm{df}=$ $1,19, P<.01)$, and UV chroma $(F=11.58, \mathrm{df}=1,19$, $P<.01)$. In contrast, background reflectance remained unaffected by the incorporation of carotenoids $(F=0.77$, $\mathrm{df}=1,19, P=.39)$.

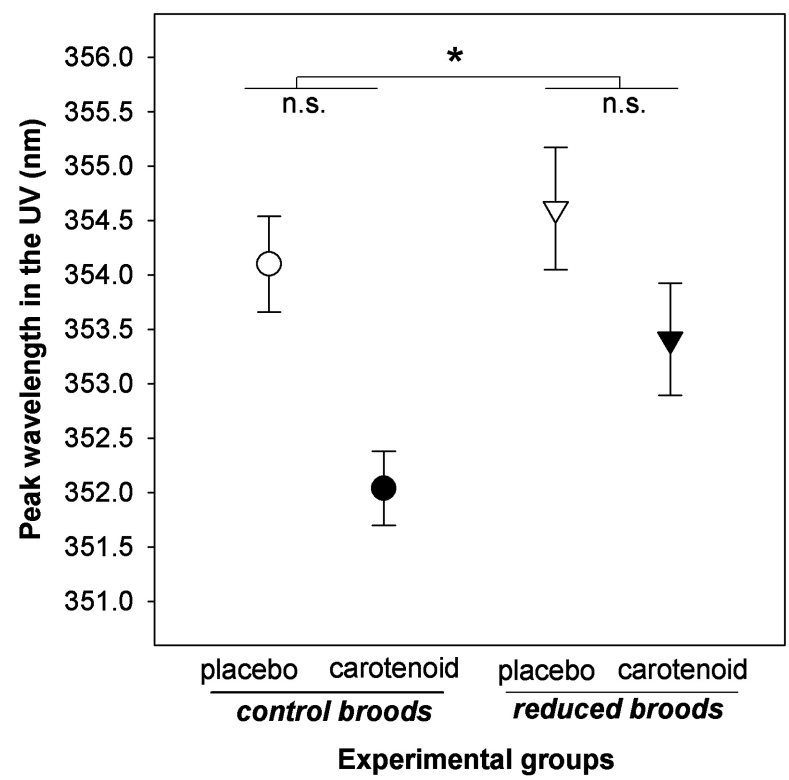

Figure 4: Effects of the carotenoid supplementation on the wavelength with the highest ultraviolet reflectance. Graph shows means and standard errors of the raw data, whereas the in-text statistics are based on restricted maximum likelihood mixed models. 


\section{Discussion}

Here, we provide experimental evidence that yellow plumage coloration of great tit nestlings consists of the summed effects of structural and pigment-based components, both being affected by environmental factors. We demonstrate in a wild bird population that environmental factors experienced during the nestling period, namely, early growth conditions and carotenoid availability, contribute independently to variation in yellow plumage coloration. While early growth conditions affected the overall background reflectance of the plumage, the carotenoid availability affected the shape-that is, a chromatic component of the reflectance curves-in both the human-visible and the UV-spectral ranges. The numerical simulations and the carotenoid removal experiment strengthen these findings. The results of both studies confirm that carotenoid incorporation affects the chromatic component of the reflectance curves in both the human-visible and the UVspectral ranges but does not affect the overall structurerelated reflectance. This is the first study demonstrating that environment-induced variation in background reflectance contributes significantly to pigment-based plumage coloration.

The experimental supplementation of carotenoids during moult is known to affect chromatic color components of yellow feathers (Hadfield and Owens 2006; Shawkey et al. 2006). Carotenoids absorb light in the short to medium wavelength, and the incorporation of lutein and zeaxanthin into the feather matrix leads to a significant reduction of reflected light in the blue wavelength and to a lesser degree also in the ultraviolet part of the spectrum. Our simulation approach and the carotenoid removal experiment confirm that the incorporation of carotenoids reduces the amount of reflected light in the ultraviolet range of the spectrum and additionally leads to a shift of the UV peak toward shorter wavelengths. Both of our findings are in line with the results from our experimental study and indicate that any treatment-induced variation in UV reflectance can be explained by variation in the carotenoid content of a feather and to a lesser degree by structural components (Jacot and Kempenaers 2007). Our results contrast to a recent study of American goldfinches, where carotenoid supplementation led to minor, nonsignificant changes in the UV range (Shawkey et al. 2006). However that study was based on a rather small sample size $(N=48)$, which may partly explain the nonsignificant treatment effects. We experimentally show that our variable background reflectance allows estimating the reflectance of the background structure independently of a feather's carotenoid content. In addition, the variable absolute carotenoid chroma was better in estimating a feather's carotenoid content than was the violet-blue chroma. The carotenoid removal explained more variance in absolute carotenoid chroma (85.3\%) than in violet-blue chroma $(78.3 \%)$. This is because absolute carotenoid chroma controls directly for background reflectance and not for overall brightness that in itself depends on carotenoid content. Our two independent measures of background structure (background reflectance) and carotenoid content (absolute carotenoid chroma) thus allow interpreting plumage colors according to the underlying biological principles responsible for the coloration.

In contrast to the effects of carotenoids on chromatic color components, the supplementation of carotenoids did not translate into reduced plumage brightness as measured by background reflectance. This finding was confirmed by both the simulation study and the carotenoid removal experiment. The brood size manipulation, that is, overall early growth conditions, had a significant effect on the amount of light reflected by the background structure. Several non-mutually exclusive mechanisms could be responsible for the observed treatment-induced variation in structural feather reflectance. While interspecific variation of white plumage colors has been attributed to diverse morphologies of the keratin layer and its incorporated air vacuoles (Prum 2006), it is possible that intraspecific variation may also reflect condition-related variation of the keratin/air matrix; however, evidence is currently lacking. Alternatively, feather brightness could result from differences in growing conditions directly related to the number of nestlings within a brood. For example, enhanced sibling competition in larger broods (Neuenschwander et al. 2003) could promote the abrasion of the feather's microstructure.

An important task for future work is to investigate the importance of carotenoid-based and structural color components in social and sexual decision-making processes. Only behavioral experiments will provide clear evidence that structure- and pigment-related color components are used by receivers. Even though chromatic and achromatic color information is perceived with different photoreceptors (chromatic color component: ultraviolet-, short-, medium-, and long-wave-sensitive receptors; achromatic color component: one double cone; Vorobyev and Osorio 1998), it is likely that different color components are integrated into one perceived signal. The integration of multiple condition-dependent color components into one signal will reinforce the reliability of the signal, where individuals who experienced beneficial conditions during moult will increase in sexual or social attractiveness. This scenario delivers an explanation of how receivers' preferences for condition-dependent signals can lead to complex multicomponent signaling systems. 


\section{Acknowledgments}

We would like to thank L. Gill for measuring the length $\rightarrow$ and coloration of great tit feathers. In addition, we thank four anonymous reviewers for helpful comments on the manuscript and H. Milewski for developing software to visualize, summarize, and analyze spectral data. The experiment was financially supported by the Swiss National Science Foundation (grant 31-53956.98 to H.R.) and conducted under a license provided by the Ethical Committee of the Office of Agriculture of the Canton of Bern, Switzerland. P.S.F. was supported by the Spanish Ministry of Education and Science (CGL2008-01522 and Programa Ramón y Cajal). B.T. is supported by the Australian Research Council (DP0879313).

\section{Literature Cited}

Andersson, S., and M. Prager. 2006. Quantifying colors. Pages 4189 in G. E. Hill and K. J. McGraw, eds. Bird coloration. Vol. 1. Harvard University Press, Cambridge, MA.

$\rightarrow$ Baayen, R. H., D. J. Davidson, and D. M. Bates. 2008. Mixed-effects modeling with crossed random effects for subjects and items. Jour nal of Memory and Language 59:390-412.

$\rightarrow$ Baker, R. R., and G. A. Parker. 1979. Evolution of bird coloration. Philosophical Transactions of the Royal Society B: Biological Sciences 287:63-130.

Bates, D., and D. Sarkar. 2006. lme4: linear mixed-effects models using S4 classes. R package, version 0.9975-9. R Foundation for Statistical Computing, Vienna.

$\rightarrow$ Botev, I. 1979. A new conception of Bouguer-Lambert-Beer's law. Fresenius' Journal of Analytical Chemistry 297:419.

$\rightarrow$ Eeva, T., E. Lehikoinen, and M. Ronka. 1998. Air pollution fades the plumage of the great tit. Functional Ecology 12:607-612.

$\rightarrow$ Fitze, P. S., B. Tschirren, and H. Richner. 2003. Carotenoid-based colour expression is determined early in nestling life. Oecologia (Berlin) 137:148-152.

Fox, J. 2002. An R and S-PLUS companion to applied regression Sage, Thousand Oaks, CA.

$\rightarrow$ Grafen, A. 1990. Biological signals as handicaps. Journal of Theoretical Biology 144:517-546.

$\rightarrow$ Hadfield, J. D., and I. P. F. Owens. 2006. Strong environmental de termination of a carotenoid-based plumage trait is not mediated by carotenoid availability. Journal of Evolutionary Biology 19 . $1104-1114$.

Hill, G. E. 2006. Female mate choice for ornamental coloration. Pages 137-200 in G. E. Hill and K. J. McGraw, eds. Bird coloration. Vol 1. Harvard University Press, Cambridge, MA.

$\rightarrow$ Jacot, A., and B. Kempenaers. 2007. Effects of nestling condition on UV plumage traits in blue tits: an experimental approach. Behavioral Ecology 18:34-40.

McGraw, K. J. 2006. Mechanics of carotenoid-based coloration. Pages
177-242 in G. E. Hill and K. J. McGraw, eds. Bird coloration. Vol. 1. Harvard University Press, Cambridge, MA.

McGraw, K. J., and A. J. Gregory. 2004. Carotenoid pigments in male American goldfinches: what is the optimal biochemical strategy for becoming colourful? Biological Journal of the Linnean Society 83:273-280.

Neuenschwander, S., M. W. G. Brinkhof, M. Kolliker, and H. Richner. 2003. Brood size, sibling competition, and the cost of begging in great tits (Parus major). Behavioral Ecology 14:457-462.

Partali, V., S. Liaaenjensen, T. Slagsvold, and J. T. Lifjeld. 1987. Carotenoids in food-chain studies. II. The food-chain of Parus spp. monitored by carotenoid analysis. Comparative Biochemistry and Physiology B 87:885-888.

$\rightarrow$ Peters, A., A. G. Denk, K. Delhey, and B. Kempenaers. 2004. Carotenoid-based bill colour as an indicator of immunocompetence and sperm performance in male mallards. Journal of Evolutionary Biology 17:1111-1120.

Prum, R. O. 2006. Anatomy, physics, and evolution of structural colors. Pages 317-319 in G. E. Hill and K. J. McGraw, eds. Bird coloration. Vol. 1. Harvard University Press, Cambridge, MA.

R Development Core Team. 2008. R: a language and environment for statistical computing. R Foundation for Statistical Computing, Vienna.

Shawkey, M. D., and G. E. Hill. 2005. Carotenoids need structural colours to shine. Biology Letters 1:121-124.

. 2006. Significance of a basal melanin layer to production of non-iridescent structural plumage color: evidence from an amelanotic Steller's jay (Cyanocitta stelleri). Journal of Experimental Biology 209:1245-1250.

$\rightarrow$ Shawkey, M. D., G. E. Hill, K. J. McGraw, W. R. Hood, and K. Huggins. 2006. An experimental test of the contributions and condition dependence of microstructure and carotenoids in yellow plumage coloration. Proceedings of the Royal Society B: Biological Sciences 273:2985-2991.

$\rightarrow$ Siefferman, L., and G. E. Hill. 2005. Evidence for sexual selection on structural plumage coloration in female eastern bluebirds (Sialia sialis). Evolution 59:1819-1828.

Tanner, M., and H. Richner. 2008. Ultraviolet reflectance of plumage for parent offspring communication in the great tit (Parus major). Behavioral Ecology 19:369-373.

Tschirren, B., P. S. Fitze, and H. Richner. 2003. Proximate mechanisms of variation in the carotenoid-based plumage coloration of nestling great tits (Parus major L.). Journal of Evolutionary Biology 16:91-100.

. 2005. Carotenoid-based nestling coloration and parental favouritism in the great tit. Oecologia (Berlin) 143:477-482.

Vorobyev, M., and D. Osorio. 1998. Receptor noise as a determinant of colour thresholds. Proceedings of the Royal Society B: Biological Sciences 265:351-358.

$\rightarrow$ Zahavi, A. 1975. Mate selection: selection for a handicap. Journal of Theoretical Biology 53:205-214.

Associate Editor: Robert Dudley Editor: Ruth G. Shaw 